\title{
Integral Sliding Mode Altitude Control for a Small Model Helicopter with Ground Effect Compensation
}

\author{
Kenichiro Nonaka and Hirokazu Sugizaki
}

\begin{abstract}
In this paper, a robust altitude control for small $\mathrm{RC}$ helicopters near ground surface is proposed. Stable takeoff, landing and hovering near surface are realized by both ground effect compensation and robust sliding mode control which suppresses the modeling error of dynamics and ground effect. To prevent the steady state error induced by the boundary layer which is indispensable to avoid chattering phenomena, integral sliding mode function is introduced which achieves asymptotic convergence to the desired altitude with continuous control input. We verified the robustness and effectiveness of the proposed control method through experiments of a RC small scale helicopter on hovering control near ground surface and external disturbance attenuation.
\end{abstract}

\section{INTRODUCTION}

Since helicopters have the capability of hovering, vertical takeoff / landing, horizontal omnidirectional motion which fixed-wing aircrafts do not have, they have been used in various fields like transport, air photography, rescue, etc [1], [2]. In recent days, unmanned helicopters equipped with navigation system and sensors come into wide use for pesticide applicator, survey and observer [3]. In order to assure safety and to increase reliability of operation, automatic control of helicopters has been widely studied by many researchers on various topics [4], [5]. Comparing with the flight altitude of fixed-wing aircrafts, that of rotary-wing airplane is much lower and close to the ground; ground effect is one of the important topic [6]. Above the level surface, for example, when the helicopter descend to the altitude which is comparable length of the blade, the lift force acts on helicopter and it can hover with less power, due to the increased air pressure below the rotor [7]. On the solid level surface, ground effect usually supports the lift of the helicopter, but it has to be considered in automatic control because precise altitude control is critical for helicopter to avoid rapid uplift, to avert unexpected collision during hovering, and to make a soft landing. It should also be noted that the ground effect depends on wind, terrain, and hardness of ground surface. Thus it is also important to design robust controller to cope with the uncertainty. In [8] and [9], takeoff and landing control is studied and experimental results are reported. For altitude control, [10] uses adaptive control, while [11] and [12] use neural network based control, to deal with both unmodeled dynamics and aerodynamic disturbance from environment. [13], [14], [15] and [16] use sliding mode control to reject them.

K. Nonaka is with the Department of Mechanical Systems Engineering, Tokyo City University, 1-28-1, Tamazutsumi, Setagaya, Tokyo, Japan.

$\mathrm{H}$. Sugizaki is with the faculty of Mechanical Systems Engineering, Tokyo City University, 1-28-1, Tamazutsumi, Setagaya, Tokyo, Japan.
In this paper, for an unmanned helicopter near ground surface, both ground effect compensation and robust integral sliding mode control are applied. Ground effect compensation is comprised of experimental model of lift force of ground effect represented as a polynomial function of altitude. Sliding mode control is used to cope with the inevitable modeling error of ground effect. As will be shown later, that error necessarily satisfies matching condition of disturbance, it can be effectively suppressed by the sliding mode control. In order to avoid chattering phenomena, boundary layer which renders control input continuous is usually introduced into switching function of virtually infinite gain. On the other hand, due to the weakened gain, steady state altitude error appears. So the integral sliding surface is successfully introduced to achieve asymptotic convergence to the desired altitude. We verified the robustness and efficacy of the proposed control through experiments of a RC small scale helicopter hovering near ground surface under disturbance.

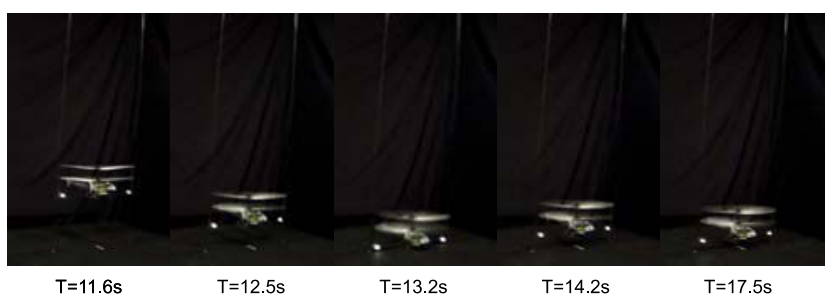

Fig. 1. Successive photograph of helicopter under ground effect

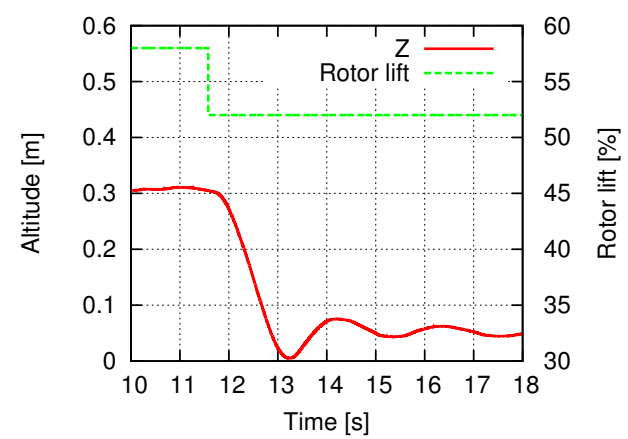

Fig. 2. Altitude and percentage of lift force

\section{GROUND EFFECT}

To show the performance change of helicopter due to ground effect of our experimental helicopter system, we first present the experimental result of a helicopter close to the ground when constant lift force generated by its rotors is decreased to smaller one. 
TABLE I

HELICOPTER SPECIFICATION

\begin{tabular}{|l|r|}
\hline Fuselage length & $360 \mathrm{~mm}$ \\
\hline Fuselage width & $90 \mathrm{~mm}$ \\
\hline Overall height & $160 \mathrm{~mm}$ \\
\hline Main rotor diameter & $350 \mathrm{~mm}$ \\
\hline Overall weight & $208 \mathrm{~g}$ \\
\hline
\end{tabular}

Fig. 1 depicts successive photograph of the helicopter near the ground when the rotor lift force was decreased from $58 \%$ to $52 \%$ of the maximum rotor lift force at $t=11.6 \mathrm{~s}$. Fig. 2 depicts the altitude and the percentage of lift force. The helicopter started to descend when lift force was decreased, but as it moved closer to the ground, additional lift force due to ground effect appeared; it hovered at the altitude close to the ground with less rotor power. Thus ground effect plays a big role in the control of helicopter near the ground. In addition, as might be expected, the terrain and wind strongly affect the performance of the helicopter. Therefore it is important to consider both ground effect compensation and robust control.

\section{EXPERIMENTAL SYSTEM}

The experimental system is discussed in this section.

\section{A. RC Helicopter}

The experimental helicopter is HIROBO XRB SR lama (Fig. 3), which is a commercially available small radio controlled helicopter with coaxial rotors which gives an advantage of inherent stability on pitching and rolling. Its specification data is shown in Table I. In order to increase the repeatability of experiments, the motion of the helicopter is constrained by two parallel wires so that it can move only in a vertical direction. Since the remaining battery capacity of this helicopter strongly affects the lift force generated by the motor, the applied lift force for the motor is modified using an empirical formula which is identified through prior experiments so that the helicopter keeps the same altitude by the constant lift force input.

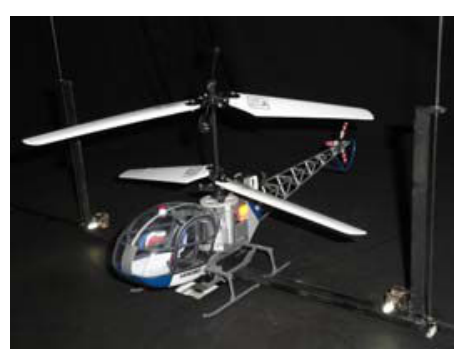

Fig. 3. Photograph of XRB SR lama with two LEDs constrained by two vertically directed parallel wires

\section{B. System Configuration}

Fig. 4 depicts the system configuration. The altitude of the helicopter is measured by the captured image of CCD camera, Sony XC-HR50. The image capture board is Photron

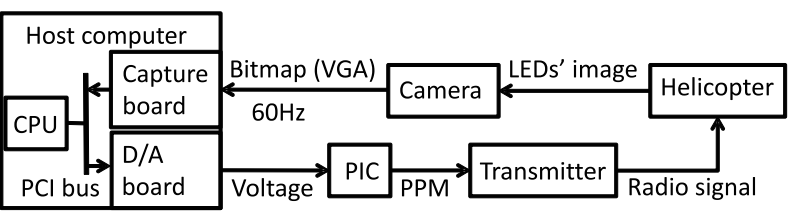

Fig. 4. Control system of RC helicopter

FDM-PCI 4TS installed in PCI bus of the host PC. This system provides $60 \mathrm{fps}$ monochrome bitmap image at $640 \times 480$ pixels (VGA) resolution. The image taken by CCD camera is transmitted through image capture board into host PC and processed to detect two LEDs attached to the helicopter.

The host PC computes the lift force of the rotor and transmit it through D/A converter and PIC into the radio transmitter to maneuver the helicopter.

\section{MODEL OF THE HELICOPTER SYSTEM}

\section{A. Dynamics of the helicopter}

The dynamics of the helicopter is given by:

$$
m \ddot{z}(t)=-m g+u(t)+F_{g}(z)+d(t)
$$

where $m[\mathrm{~kg}]$ is the mass of the helicopter, $z[\mathrm{~m}]$ is the altitude, $m g[\mathrm{~N}]$ is the gravitational force, $u[\mathrm{~N}]$ is the lift force generated by rotors, $F_{g}(z)[\mathrm{N}]$ is the nominal lift force due to ground effect, $d[\mathrm{~N}]$ is the unknown disturbance including modeling error of the ground effect. Even though $d(t)$ is unknown, it could be directly canceled by control input $u(t)$ when $u(t)=-d(t)$. Thus $d(t)$ satisfies the matching condition on which SMC works effectively.

\section{B. Model of Ground Effect}

Since it is difficult to get the analytical model of the ground effect, we use an empirical formula which is identified through hovering experiments at several altitudes. Fig. 5 depicts the voltages of rotors $f_{g}[\%]$ which renders the helicopter hover at each altitudes $z[\mathrm{~m}]$. Since data points draw a smooth curve, we approximate the percentage for rotor lift as a 2 nd-order polynomial function $f_{g}(z)$ given by

$$
f_{g}(z)=-78.2 z^{2}+63.1 z+47.3
$$

using least squares approximation. This model is used from $0 \mathrm{~mm}$ to $400 \mathrm{~mm}$. For the altitude above $400 \mathrm{~mm}, f_{g}(z)$ is set to be a constant value $f_{g}(400 \mathrm{~mm})$.

To describe ground effect in terms of force, we also identified an empirical formula between rotor lift percentage and lift force by adding $3 \mathrm{~g}, 6 \mathrm{~g}, 9 \mathrm{~g}, 12 \mathrm{~g}$ and $15 \mathrm{~g}$ weights, at the altitude $800 \mathrm{~mm}$ where the helicopter is out of ground effect. The experimental result is depicted in Fig. 6 where $F_{t}[\mathrm{~N}]$ represents the net lift force. Using least squares approximation, the empirical formula of $F_{t}[\mathrm{~N}]$ is given by

$$
F_{t}=0.031 \times(\text { Rotor lift }[\%])+0.283 \text {. }
$$

Plugging (2) into (3) for rotor lift percentage, $F_{g}(z)$ in (1) is represented by

$$
F_{g}(z)=0.031 f_{g}(z)+0.283 .
$$




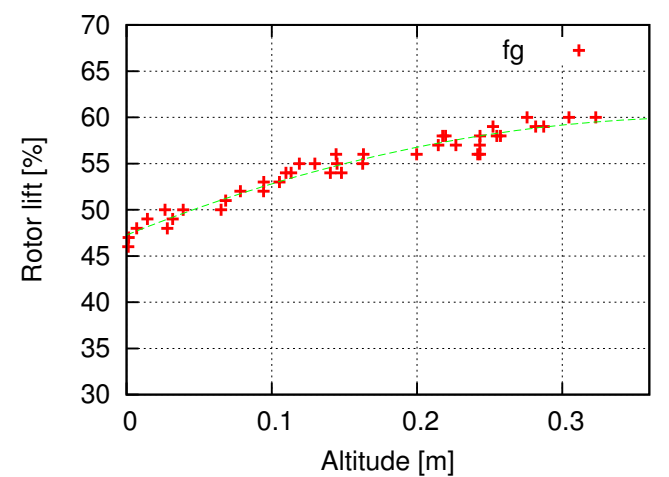

Fig. 5. Rotor lift percentage required to hover at each altitude

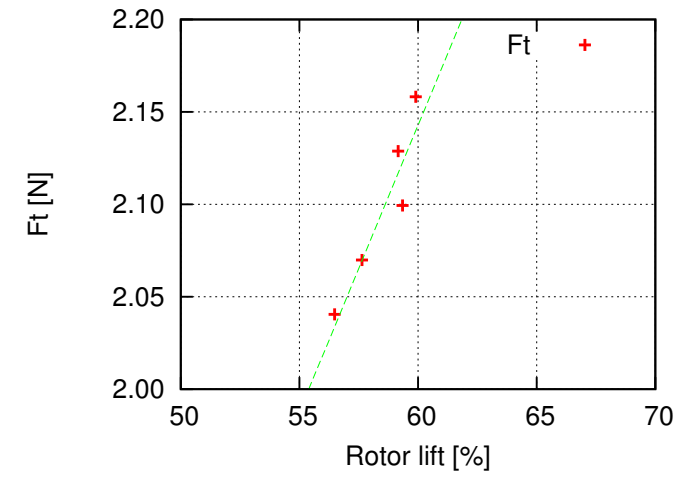

Fig. 6. Lift force for rotor lift percentage

\section{Integral SLiding Mode Controller}

In the previous section, the empirical model of $F_{g}(z)$ for (1) is derived. Since the experimental data indicates that the modeling error of $F_{g}(z)$ is bounded, unknown disturbance $d(t)$ of (1) is also bounded. In this section, together with the reference model, sliding mode controller for (1) which suppresses the effect of $d(t)$ is presented.

\section{A. Reference Model}

The target altitude of the unmanned helicopter may vary discontinuously; we use the following 3rd order lag system to generate reference altitude $z_{r}(t)$ from target altitude $z_{t}(t)$ :

$$
\frac{Z_{r}(s)}{Z_{t}(s)}=\frac{1}{T s+1} \cdot \frac{\omega_{n}^{2}}{s^{2}+2 \zeta \omega_{n} s+\omega_{n}^{2}}
$$

where $Z_{r}(s):=\mathcal{L}\left[z_{r}(t)\right]$ and $Z_{t}(s):=\mathcal{L}\left[z_{t}(t)\right]$ respectively. The relative degree of (5) has to be greater than 2, because $\dot{z}_{r}(t)$ and $\ddot{z}_{r}(t)$ is required for sliding mode controller shown in the following section.

\section{B. Integral Sliding Mode Control}

Let $e(t):=z(t)-z_{r}(t)$ be the tracking error for the reference altitude. Let $\sigma(t) \equiv 0$ be the sliding surface. $\sigma(t)$ is defined by

$$
\sigma(t)=\dot{e}(t)+\alpha e(t)+\lambda \eta(t)
$$

where

$$
\eta(t):=\int_{0}^{t} e(\tau) \mathrm{d} \tau
$$

It should be noted that the sliding mode dynamics $\sigma(t) \equiv 0$ is asymptotically stable if and only if $\alpha>0$ and $\lambda>0$.

To derive a sliding mode controller for $u(t)$, taking first derivative of $\sigma(t)$ with respect to $t$ and plugging (1) into it, we get

$$
\begin{aligned}
\dot{\sigma}(t)= & \ddot{e}(t)+\alpha \dot{e}(t)+\lambda \dot{\eta}(t) \\
= & m^{-1}\left(-m g+u(t)+F_{g}(z)+d(t)\right) \\
& -\ddot{z}_{r}(t)+\alpha \dot{e}(t)+\lambda e(t) .
\end{aligned}
$$

The sliding mode control input is usually designed so that the sliding mode $\sigma(t) \equiv 0$ is achieved in finite time. In this system, control input $u(t)$ is given by

$$
\begin{aligned}
u(t)= & m g-F_{g}(z)+m\left(\ddot{z}_{r}(t)-\alpha \dot{e}(t)-\lambda e(t)\right) \\
& -\Gamma \operatorname{sgn}(\sigma(t))
\end{aligned}
$$

where $\Gamma$ is a sufficiently large positive constant which satisfies

$$
\Gamma>\sup _{t}|d(t)|
$$

and sgn is a signum function:

$$
\operatorname{sgn}(\sigma(t))=\left\{\begin{array}{rl}
+1 & (\sigma(t)>0) \\
0 & (\sigma(t)=0) \\
-1 & (\sigma(t)<0)
\end{array} .\right.
$$

Plugging (9) into (8), we get

$$
m \dot{\sigma}(t)=d(t)-\Gamma \operatorname{sgn}(\sigma(t)),
$$

which indicates that $\sigma(t) \equiv 0$ is achieved in finite time. As mentioned before, the sliding mode dynamics $\sigma(t) \equiv 0$ is asymptotically stable so that $e(t)$ asymptotically converges to zero; $z(t)$ asymptotically tracks $z_{r}(t)$ regardless of $d(t)$.

We make a realistic assumption that $\Gamma$ which satisfies (10) is known in this paper. For $d(t)$ with unknown upper bound, application of adaptive estimation is promising.

\section{Boundary layer and its stability analysis}

Since signum function (11) requires infinitesimal switching time, it often causes chattering phenomena. To avert it, (11) is approximated by a saturation function given by

$$
\operatorname{sat}(\sigma(t))=\left\{\begin{array}{cc}
+1 & (\sigma(t)>+\epsilon) \\
\sigma / \epsilon & (|\sigma(t)| \leq \epsilon) \\
-1 & (\sigma(t)<-\epsilon)
\end{array}\right.
$$

where $\epsilon$ is a small positive constant indicating half width of boundary layer. As shown below, the smaller $\epsilon$ is, the tracking performance becomes better. In particular, (13) for $\epsilon=0$ is identical with (11), where perfect rejection of $d(t)$ is achieved. But smaller $\epsilon$ increases strain of actuators, $\epsilon$ should be tuned properly depending on the response capability of the rotor motors.

(9) is then represented as

$$
\begin{aligned}
u(t)= & m g-F_{g}(z)+m\left(\ddot{z}_{r}(t)-\alpha \dot{e}(t)-\lambda e(t)\right) \\
& -\Gamma \operatorname{sat}(\sigma(t)) .
\end{aligned}
$$

Plugging (14) into (8), we get

$$
m \dot{\sigma}(t)=d(t)-\Gamma \operatorname{sat}(\sigma(t))
$$


which indicates that $|\sigma(t)| \leq \epsilon$ is achieved in finite time. To derive stability condition, let us consider the case, $|\sigma(t)| \leq \epsilon$. (15) can be written by

$$
m \dot{\sigma}(t)=d(t)-\frac{\Gamma}{\epsilon} \sigma(t) .
$$

Taking Laplace transformation of (6) and (16) and deleting $\sigma$, we get the transfer function from $d(t)$ to $e(t)$ :

$$
\frac{E(s)}{D(s)}=\frac{s}{s^{2}+\alpha s+\lambda} \cdot \frac{\epsilon}{\epsilon m s+\Gamma}
$$

where $E(s):=\mathcal{L}[e(t)]$ and $D(s):=\mathcal{L}[d(t)]$. This transfer function indicates that $d(t)$ does affect tracking error $e(t)$ for $\epsilon \neq 0$, but sufficient attenuation is possible for proper choice of parameters $\alpha, \lambda, \Gamma$, and $\epsilon$. Especially, for DC component of $d(t)$, asymptotic attenuation is achieved.

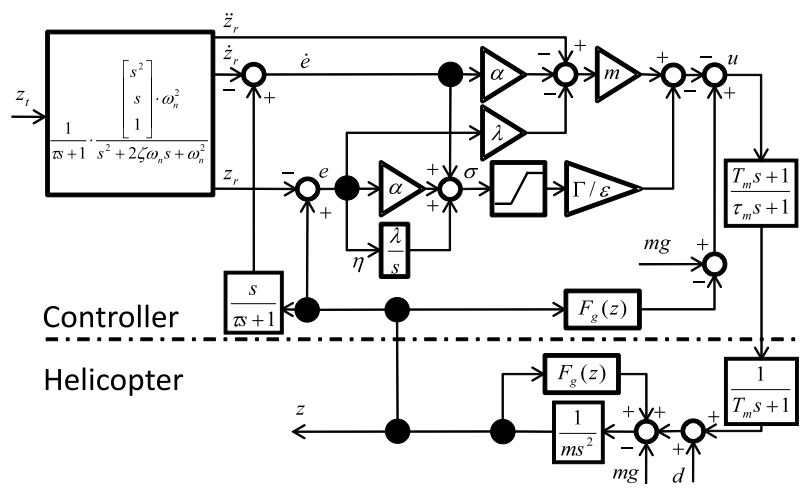

Fig. 7. Block diagram of control system

It should be noted that if $\lambda=0$, (6) becomes a sliding mode function without integrator,

$$
\sigma(t)=\dot{e}(t)+\alpha e(t)
$$

which induces asymptotically stable convergence: $z(t) \rightarrow$ $z_{r}(t)$ when $\sigma(t) \equiv 0$ for $\alpha>0$. In practice, (14) with $\epsilon \neq 0$ is used to avoid infinitesimal chattering; then the transfer function from $d(t)$ to $e(t)$ of (17) becomes

$$
\frac{E(s)}{D(s)}=\frac{1}{s+\alpha} \cdot \frac{\epsilon}{\epsilon m s+\Gamma}
$$

which brings in steady state error for $d(t)$ with DC component. Thus the integrator is useful to reject constant disturbance.

The block diagram of the whole system is depicted in Fig. 7. There is a lag element of the rotor which is approximated by $1 \mathrm{st}$ order lag element $1 /\left(T_{m} s+1\right)$. Lead filter $\left(T_{m} s+1\right) /\left(\tau_{m} s+1\right)$ is applied to $u(t)$ to compensate it.

\section{EXPERIMENTAL RESULTS}

To demonstrate the efficacy of the proposed controller, we show two experiments: the first one is tracking control of the altitude which varies stepwise by SMC with the ground effect compensation and without it. The second one is to apply external constant disturbance during hovering by appending a small weight to the helicopter.

\section{A. Tracking Control for Stepwise Altitude}

The target altitude $z_{t}(t)$ is increased by $200 \mathrm{~mm}$ every $15 \mathrm{~s}$ until it reaches $600 \mathrm{~mm}$, then the target altitude $z_{t}(t)$ is decreased by $200 \mathrm{~mm}$ every $15 \mathrm{~s}$ until it reaches $0 \mathrm{~mm}$. For the reference model (5), parameters are chosen to be $T=1 \mathrm{~s}, \omega_{n}=2 \pi \mathrm{rad} / \mathrm{s}$ and $\zeta=1$ with consideration for the time constant of rotor motors. For the integral sliding mode controller (14), designed parameters are $\alpha=1.55, \epsilon=$ 0.2 and $\Gamma=0.312$ where $\Gamma$ satisfies (10) for the expected modeling error of the ground effect estimated from Fig. 5 and Fig. 6. In this experiment, we set $\lambda=0$ to see the efficacy of ground effect compensation. Parameters for the phase-lead filter are $\tau=0.05 \mathrm{~s}, \tau_{m}=0.002 \mathrm{~s}$ and $T_{m}=0.2 \mathrm{~s}$.

The experimental results of SMC without ground effect compensation is depicted in Fig. 8 for $z_{r}(t)$ and $z(t)$, Fig. 9 for the rotor lift force percentage, and Fig. 10 for $\sigma(t)$ with the width of boundary layer respectively. $f_{g}(z)$ is set to be a constant value $f_{g}(0.4)$.

The experimental results of SMC with ground effect compensation is depicted in Fig. 11 for $z_{r}(t)$ and $z(t)$, Fig. 12 for the rotor lift force percentage, and Fig. 13 for $\sigma(t)$ with the width of boundary layer respectively.

Fig. 8 indicates that large deviation appears due to ground effect near ground, but Fig. 11 shows that the tracking performance is improved by compensating the ground effect. When the altitude is out of ground effect $(z=400 \mathrm{~mm}$ or $600 \mathrm{~mm}$ ), the tracking performance without ground effect compensation differ little from the one with compensation. But as the altitude descends, the tracking performance of the former one is deteriorated and the altitude is kept high because of the increased ground effect.

\section{B. Disturbance Attenuation}

To see the disturbance attenuation of the integral SMC, we applied an external constant force, $d=-118 \mathrm{mN}$, from $t=15 \mathrm{~s}$ to $t=30 \mathrm{~s}$ by appending a $12.0 \mathrm{~g}$ weight to the helicopter. To use the integrator, we set $\lambda=0.707$ while other control parameters are the same with the previous ones. The maximum gain of the transfer function (17) is $-7.7 \mathrm{~dB}$, about the same as that of (19), so that the disturbance attenuation capability is unchanged while asymptotic rejection of constant disturbance is achieved.

Fig. 14, Fig. 15, and Fig. 16 depict the experimental results of SMC with $\lambda=0$ for $\left(z_{r}(t), z(t)\right), u(t)$ and $\sigma(t)$ respectively, while Fig. 17, Fig. 18, and Fig. 19 depicts the experimental results of Integral SMC. In both controller, successful takeoff and landing were achieved. As depicted in Fig. 14, persistent deviation due to external disturbance appeared in SMC, but for integral SMC depicted in Fig. 17, $z(t)$ asymptotically converges to $z_{r}(t)$ due to the integrator.

\section{CONCLUSION}

In this paper, a robust altitude control for small RC helicopters near ground surface is proposed. Stable takeoff, landing and hovering near surface are realized by both ground effect compensation and robust sliding mode control which suppresses the modeling error of ground effect 


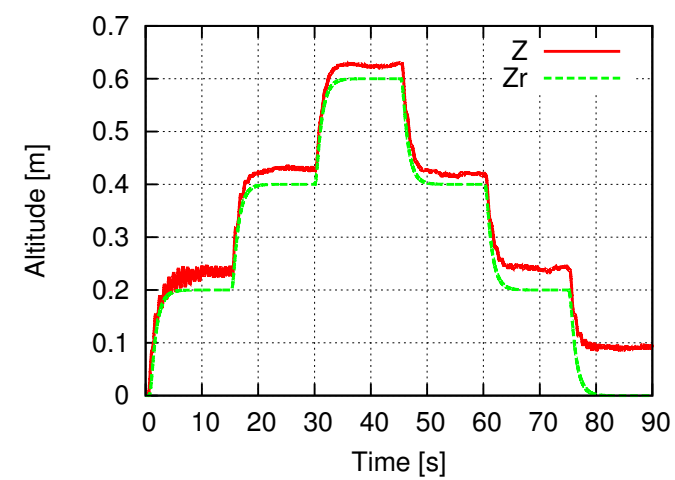

Fig. 8. $\quad z_{r}$ and $z$ (without ground effect compensation)

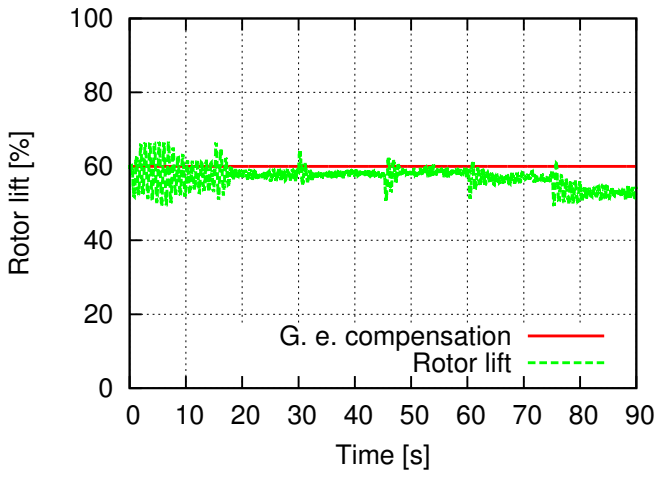

Fig. 9. Rotor lift percentage (without ground effect compensation)

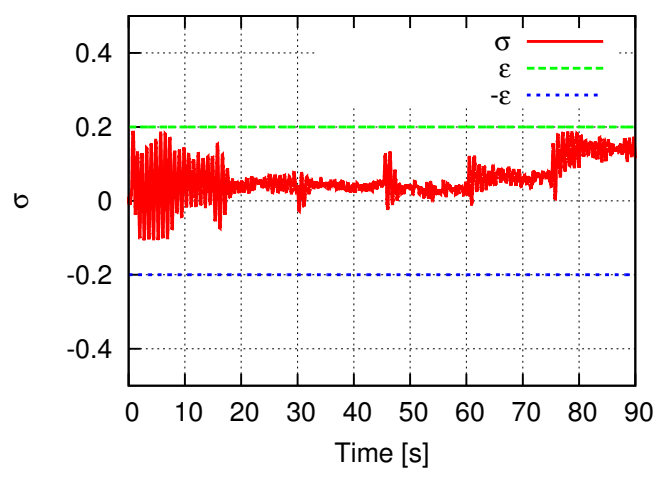

Fig. 10. $\sigma$ (without ground effect compensation)

and external disturbance. To prevent the steady state error induced by the boundary layer which is indispensable to avoid chattering phenomena, integral sliding mode function is introduced which achieves asymptotic convergence to the desired altitude with continuous control input. We verified the robustness and effectiveness of the proposed control method through experiments of a RC small scale helicopter on hovering control near ground surface and external disturbance attenuation.

\section{ACKNOWLEDGMENTS}

The authors gratefully acknowledge the contribution of helpful comments from Prof. Katsumasa Suzuki of Tokyo City University.

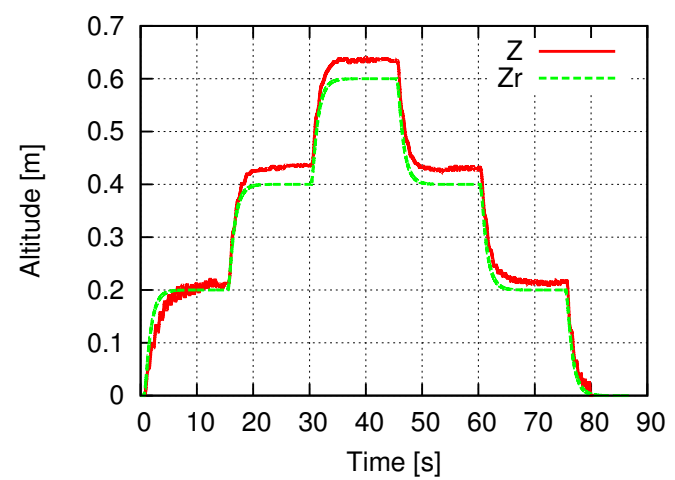

Fig. 11. $z_{r}$ and $z$ (with ground effect compensation)

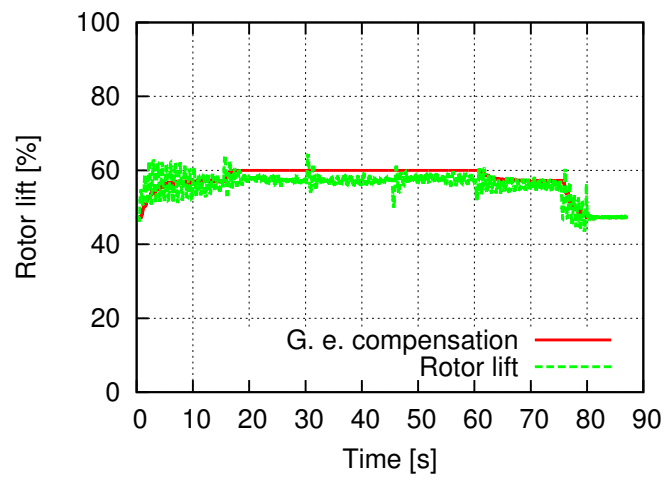

Fig. 12. Rotor lift percentage (with ground effect compensation)

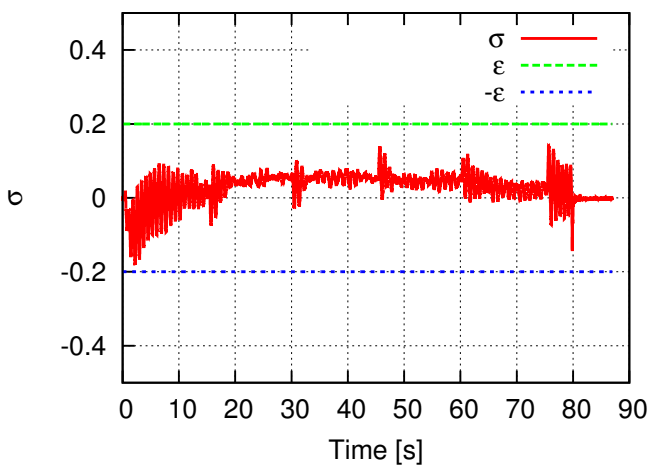

Fig. 13. $\sigma$ (with ground effect compensation)

\section{REFERENCES}

[1] Hideki Shibata, Development of Autonomous unmanned Helicopter "RMAX-G1", Journal of the Japan Society for Aeronautical Space Sciences, Vol. 54, No. 628, pp.140-144, 2006.

[2] Kenzo Nonami, Rotary Wing Aerial Robotics, Journal of the Robotics Society of Japan, Vol. 24, No. 8, pp.890-896, 2006.

[3] Hiroaki Nakanishi, Observation Activities at Disaster Sites Using an Autonomous Unmanned Helicopter, Japan Society of Mechanical Engineers, Vol. 112, No. 1091, pp. 45-48, 2009.

[4] Ryosuke Mori, Kenichi Hirata, Takuya Kinoshita, Vision-Based Guidance Control of a Small-Scale Unmanned Helicopter, Proceedings of the 2007 IEEE/RSJ International Conference on Intelligent Robotics and Systems, pp.2648-2653, 2008.

[5] Kei Watanabe, Yasushi Iwatani, Kenichiro Nonaka, and Koichi Hashimoto. A visual-servo-based assistant system for unmanned helicopter control. In IEEE/RSJ International Conference on Intelligent Robots and Systems, pp. 822-827, Nice, France, September 2008.

[6] Xiaodong Wang and Xiaoguang Zhao, A practical survey on the flight control system of small-scale unmanned helicopter, 7th World 


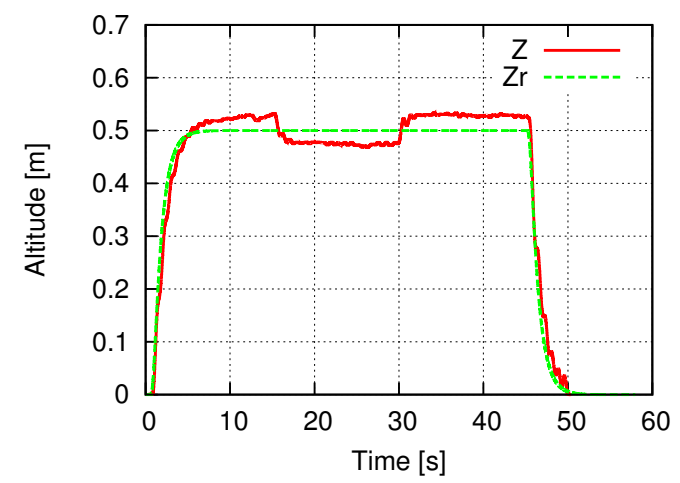

Fig. 14. $z_{r}$ and $z(\mathrm{SMC})$

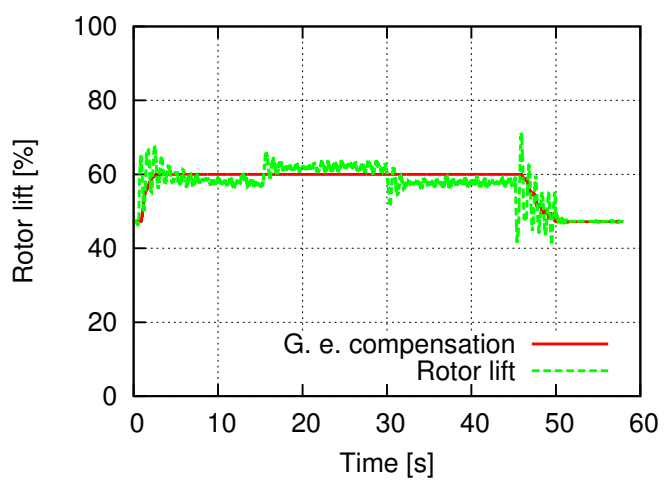

Fig. 15. Rotor lift percentage and grand effect compensation included (SMC)

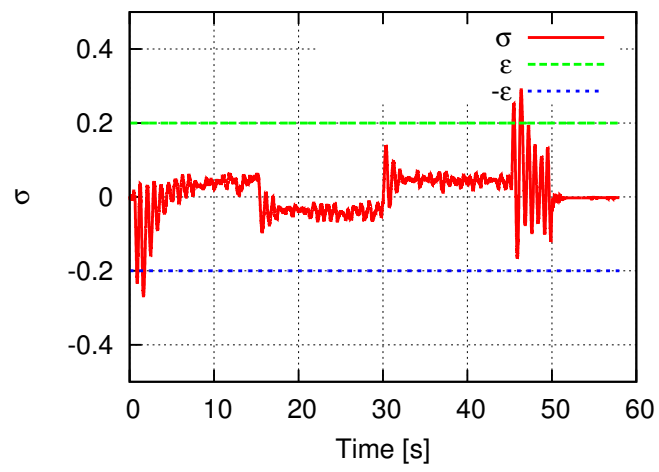

Fig. 16. $\sigma$ (SMC)

Congress on Intelligent Control and Automation (WCICA 2008), pp.364-369, 2008.

[7] Japan Aeronautical Engineers' Association, Helicopter Handbook, Japan Aeronautical Engineers' Association, (1981),

[8] Yasushi Iwatani, Kei Watanabe, and Koichi Hashimoto. Automatic take-off and landing of an unmanned helicopter using vision-based control with occlusion handling. In 17th CISM-IFToMM Symposium on Robot Design, Dynamics, and Control (RoManSy), pp. 11-18, Tokyo, Japan, July 2008.

[9] Masanori Fujita, Akira Shimada, Takeoff and Landing Control using Force sensor by Electrically-Powered Helicopters, Journal of the Institute of Electrical Engineers of Japan, Vol.127, No.2, pp.112117, 2007.

[10] A. Dzul, R. Lozano, P. Castillo, Adaptive altitude control for a small helicopter in a vertical flying stand Proceedings of the 42nd IEEE Conference on Decision and Control, pp. 2710-2715, 2003.

[11] K.P.Tee, S.S.Ge, and F.E.H.Tay, Adaptive neural network control for helicopters in vertical flight, IEEE Transactions on Control Systems Technology, Vol. 16, No. 4, pp. 753-762, 2008.

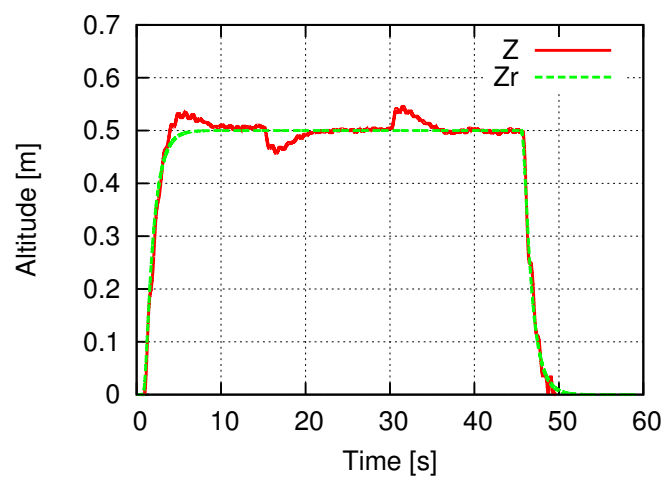

Fig. 17. $z_{r}$ and $z$ (Integral SMC)

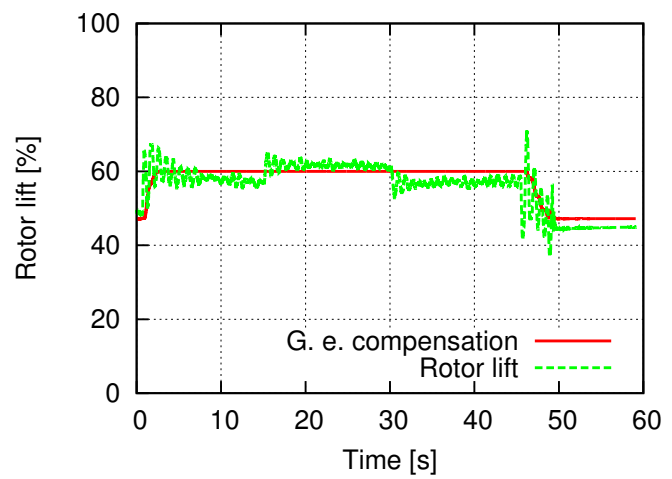

Fig. 18. Rotor lift percentage and grand effect compensation included (Integral SMC)

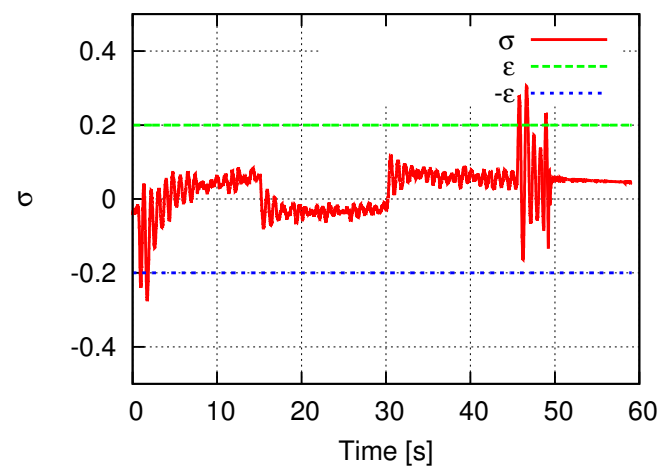

Fig. 19. $\sigma$ (Integral SMC)

[12] S.S.Ge, B.Ren, K.P.Tee, and T.H.Lee, Approximation based control of uncertain helicopter dynamics, IET Control Theory and Applications, Vol. 3, No.9, pp. 941-956, 2009.

[13] H. Sira-Ramirez, M. Zribi, S. Ahmad, Dynamical sliding mode control approach for vertical flight regulation in helicopters IEE Proceedings of Control Theory and Applications, Vol. 141, Issue 1, pp.19-24, 1994.

[14] Takahisa Nagata and Kenichiro Nonaka, Altitude Control experiment of RC helicopter by SMC near ground surface, The 51th Japan Joint Automatic Control Conference, pp. 403-406, 2008

[15] A. Martini, F. Leonard, G. Abba, Robust nonlinear control and stability analysis of a 7DOF model-scale helicopter under vertical wind gust, IEEE/RSJ International Conference on Intelligent Robots and Systems (IROS 2008) pp. 354-359, 2008.

[16] M.O.Efe, Robust low altitude behavior control of a quadrotor rotorcraft through sliding modes Mediterranean Conference on Control and Automation, pp. 1-6, 2007. 